\title{
Subject Index Vol. 16, 1996
}

Absolute risk 182 Acetazolamide 315 N-Acetyl-ß-D-glucosaminidase 300 Acidosis 382 Acute allograft rejection 273

- $\quad$ renal failure $154,162,532$

Adequacy 29, 35

Adriamycin nephropathy 540

AIDS 446

Ambient temperature 375 Ambulatory blood pressure 190

-

Amyloid 114

- - monitoring 202

Amyloidosis 149

Anemia 268

Angiokeratoma corporis diffusum 352 Antiglomerular basement membrane antibodies 442

Antihypertensives 210,223,237 -, assessment 202

Antimicrosomal thyroid antibodies 478 Antineutrophil cytoplasm antibodies 442 Anti-neutrophil-

cytoplasmic-antibody

serology 106 Antinuclear antibodies 478 Apolipoprotein AI 287

- B 287

Arginine vasopressin 513 Arterial compliance 217 Arteriovenous fistula 118

- $\quad$ graft 118

AST-120 124

Atrial natriuretic peptide(s) 128, 462 Autoimmunity 478

Bipolar disorder 339

Blood pressure 35, 182,217,263

- determination 190

- monitors 190

volume 513 Bromide poisoning 537

Calciphylaxis 344

Calcitriol 73

Cancer 402

Cardiac output 263

Carnitine palmitoyltransferase 162

Castration, male rats 540

CD10 antigen 273

Central diabetes insipidus 339

- $\quad$ hypervolemia 128

Chloride 462, 537

Cholesteryl ester transfer protein 394

Chronic hepatitis C 478

renal disease 471 
- failure 287, 394,489

- insufficiency 138 Circadian rhythm(s) 202, 462 Clinical effects 263 Coagulation time 309

Compartment analysis 17 Complement activation 293 Coronary heart disease 210

hypothesis 210 Cost-effectiveness 202 51Cr-EDTA 500 Creatinine clearance 124 Cryoglobulinemia 159

Delivery 29 Dextran 73 Diabetes 223

mellitus 217,315 Diabetic renal disease 237 Diagnosis 190 Diagnostic problems 358 Dialysis 45, 382,386

dose 7

Dialysis-related amyloidosis 484 Dialyzer 29

Digibind 529

Digoxin-like immunoreactive factors 529

DNA sequencing 352

Dopamine 489

Dopaminergic drugs 489

Dynamic magnetic resonance imaging 506

Electron microscopy 352, 412

Enalapril 315

Endocarditis 159

Endothelin 246

Endotoxin 60

End-stage renal disease 79, 118, 344, 375

- failure 352

- -, pulmonary function 144 Erythrocyte 369 Erythropoietin 73, 304

ESRD 7

Fabry disease 352

Fe-gluconate-Na complex 268

Ferropenia 268

Fibrillary glomerulonephritis 523

Formaldehyde 52

Fourier analysis 375

Frameshift mutation 352

Free apolipoprotein E 287

- fatty acids 327

Gadolinium-DTPA 506

$\alpha$-Galactosidase 352

Gastrointestinal tract 149

Glomerular basement membrane, thin 412

filtration rate 315,471

- -, measurement 138 Glomeruli 320 Glomerulonephritis 159 Glomerulosclerosis, pathogenesis 540

Glucose metabolism 304 Glutaraldehyde advantages,

disadvantages 52 Graft biopsy 98

Hematuria, isolated 412 Hemodialysis 17,29,35,73,79,95, 118, 149,280,287,293,304,309

- $\quad$ patients, adequate body iron stores 268

Hemodialyzed patients, lipid transfers 394

Hemodynamics 210,513 
Hemolytic uremic syndrome 446 Hepatic triglyceride lipase 309 Hepatitis B vaccination 95

C virus 95 Hepatocytes 431 High-flux hemodialysis 484 HIV 446

infection 280 Hospitalization 118 Hypercoagulability 344 Hyperparathyroidism 344 Hypertension

91,190,217,223,237 Hypertriglyceridemia 394 Hypertrophy, cardiac 263 Hypocalcemia 386

Hypokalemia 369 Hyponatremia 349 Hypotensive therapy 182

Ibopamine 489

Immunoglobulin A nephropathy 412, 500

- $\quad$ therapy 532

Immunotactoid glomerulopathy 523 Inappropriate antidiuretic hormone secretion syndrome 349

Incidence 7 Insulin action 223

- $\quad$ resistance 223,304

Insulin-dependent diabetes mellitus 300

Integrins 402

558

$\alpha$-Interferon therapy 478 Interleukin-1ß 293 Intestinal permeability 500 Intracellular pH 431 Iohexol

138 Iothalamate 138 Iron 73

Kaliuretic peptide 128 Karnofsky score 118 Kidney $114,402,506$

donation 334

hypertrophy 425

transplant 98

transplantation $334 \mathrm{Kt} / \mathrm{V} 7,17$

Lactic acidosis 520 Leishmaniasis infection 358 Limulus amoebocyte lysate assay 60 Lipase 309

Lipoprotein 394

- $\quad$ lipase 309

Lithium 339

Long-term survival 455

Lymphocele 361

Magnetic resonance renogram 506

Malaria 513

Malnutrition 79

Marfan syndrome 320

Medications 73

Metformin 520

Microfibrils 320

B2-Microglobulin 149,484

Mizoribine 167

Modeling 17

Monitoring 106

Monoclonal gammopathy 523

Morbidity 386

Morphometry 412

Mortality 7, 386

Myoglobulinuria 162

$\mathrm{Na}+\mathrm{H}+$ exchanger activity $431 \mathrm{Na}, \mathrm{K}-\mathrm{ATP}$ ase 369 Nephrogenic diabetes insipidus 339 Net neutral lipid transfer 394 Neuromyopathy 114 Neutral endopeptidase 273 Nondiabetic renal disease 237

Nonvascular access 118 Norepinephrine 513 Nutritional assessment 79 
Osmotic nephrosis 532 Osteopontin 402 Outcome 98

Page kidney 91 Paraganglioma 91 Parathyroid hormone 431 Pasteurella multocida 361 Peracetic acid 52 Peripheral resistance 263

vasodilatation 252 Peritoneal clearances 455

dialysis 79, 455 Peritonitis 455 Plasma phospholipids 409

renin activity 471 Plasmapheresis 446 Platelets 409 Polyanionic sites 167 Polyarteritis 442 Polyuria 339 Potassium 369 Prediction 98 Predictors 280 Preeclampsia 529 Prognosis 98 Prognostic indicator 500 Proliferative changes 210 Propantheline bromide 537 Protein C 344

catabolic rate 382

catabolism 382 Pulmonary function, renal transplantation

144 Puromycin aminonucleoside rats 167 Pyrogenic reactions 60

Q fever 159 Quantification 17

Receptors 246

Reciprocal creatinine 124

Recirculation 29

Recombinant human erythropoietin 263

Relative risk 182

Renal allograft recipient 358

compression 91

disease 320

failure 334,520

-, progression 489

hemodynamics 252

physiology 506

transplantation 273, 358, 361, 478

-, pulmonary function 144

Renin 91,513

- $\quad$ inhibition 252

Renotropin 425

Reset osmostat syndrome 349 Reuse 52

Reverse osmosis 60 Rhabdomyolysis 162 Rheumatoid factors 478 Risk factors 210

Signaling 246

Sodium 462

Soluble ELAM-1 106

Sudden unexplained death syndrome 369

Survival 35,280

Systemic disease 523

- $\quad$ resistance 217

Thrombotic microangiopathy 446

- $\quad$ thrombocytopenic purpura 446

Thromboxane B2 167

Transplantation 114,352

Treatment time 35

Tubules 402

Tubuloglomerular feedback 315 Tubulointerstitial nephritis 154 Tumor necrosis factor- $\alpha 293$

Ultrasonography 154 Urea cycle 520 
kinetic modeling 45

kinetics 17 Uremia 327,409

Urinary albumin excretion 237 USRDS 7

Valproicacid 327

Vancomycin 73

Vascular access 29,45, 118

lesions 320

Vasculitis 106,442

Vasopressin 339

Vessels 320

Visceral leishmaniasis 358 Vitronectin 402

Water immersion 128

load 513

Yersinia pseudotuberculosis 154

Subject Index Vol. 16, 1996

559 